University of Nebraska - Lincoln

DigitalCommons@University of Nebraska - Lincoln

Other Publications in Zoonotics and Wildlife

Disease

Wildlife Disease and Zoonotics

$1-2006$

\title{
Progress in diagnosis, treatment and elimination of echinococcosis and cysticercosis
}

Peter M. Schantz

Centers For Disease Control and Prevention, Atlanta, GA, pschantz@cdc.gov

Follow this and additional works at: https://digitalcommons.unl.edu/zoonoticspub

Part of the Veterinary Infectious Diseases Commons

Schantz, Peter M., "Progress in diagnosis, treatment and elimination of echinococcosis and cysticercosis" (2006). Other Publications in Zoonotics and Wildlife Disease. 42.

https://digitalcommons.unl.edu/zoonoticspub/42

This Article is brought to you for free and open access by the Wildlife Disease and Zoonotics at DigitalCommons@University of Nebraska - Lincoln. It has been accepted for inclusion in Other Publications in Zoonotics and Wildlife Disease by an authorized administrator of DigitalCommons@University of Nebraska - Lincoln. 


\title{
Progress in diagnosis, treatment and elimination of echinococcosis and cysticercosis
}

\author{
Peter M. Schantz * \\ Division of Parasitic Diseases, Coordinating Center for Infectious Diseases, Centers For Disease Control and Prevention, Atlanta, GA 30341, USA
}

Available online 4 January 2006

\begin{abstract}
Here I review the current status of geographical occurrence and public health significance of echinococcosis (Echinococcus spp. infections) and cysticercosis (Taenia solium infection) with special emphasis on the remarkable technologic progress achieved in recent decades that has led to greater understanding of the biology and epidemiology of these cestode infections. The greatest remaining challenges are to apply this knowledge and technology to improved medical management and prevention of these infections.
\end{abstract}

Keywords: Cestodes; Tapeworms; Hydatid disease; Echinococcosis; Cysticercosis; Taeniasis

\section{Introduction}

Thank you for this opportunity to share my experiences and observations for the future in research, treatment and control of echinococcosis and cysticercosis/taeniasis. My experience is shaped by my activities directed toward these cestode parasites in the field and in the laboratory. I first encountered Taenia solium taeniasis/cysticercosis as a Fellow in Medical Parasitology at the National University in Mexico and at that time only the most serious of cases of neurocysticercosis were correctly diagnosed, often at autopsy. I first encountered echinococcosis in California when, upon visiting a local slaughterhouse to collect cysticerci of Taenia hydatigena, we noted hydatid cysts in the viscera of sheep. This led to the work that formed my PhD thesis at the University of California Davis. Upon completion of graduate study I began a job as Epidemiologist at the Pan American Health Organization's Zoonoses Research Center (CEPANZO) in Buenos Aires, Argentina. A major emphasis of our program was working with scientists at Latin American government institutions in carrying out research and implementing control programs against echinococcosis. In 1974 I had the opportunity to join the U.S. Public Health Service at the Centers for Disease Control and Prevention in Atlanta. In these positions I have had the opportunity to work with scientists from many countries in research and control of

\footnotetext{
* Tel.: +1 404488 7767; fax: +1 4044887761 .
}

E-mail address: pschantz@cdc.gov. parasitic zoonoses with special emphasis on echinococcosis and cysticercosis. It gives me pleasure to reflect upon these experiences and to marvel at just how far we have progressed in the understanding of these zoonotic infections and in development of technology that is effectively applied to diagnosis, treatment and control of these infections.

\section{Echinococcosis}

Until recently, the genus Echinococcus had contained 4 generally accepted species: Echinococcus granulosus (Batsch, 1786), Echinococcus multilocularis (Leuckart, 1863), Echinococcus oligarthrus (Diesing, 1863), and Echinococcus vogeli (Rausch and Bernstein, 1972) are morphologically distinct in both adult and larval stages [1,2].

One of these species, E. granulosus, is made up of a number of biologically and genetically distinct entities, whose taxonomic positions are in states of flux. These variants are separated ecologically, although not necessarily geographically, by the nature of their respective unique host assemblages. They have been referred to as strains or subspecies [2], however, recent proposals have elevated a number of them to separate species status. Current data, based on genome patterns, generally support previous characterizations based on morphologic and biologic criteria: at least 10 genetically distinct populations exist within the complex until recently denoted $E$. granulosus [2] (Table 1). Probes characterizing the mitochon- 
Table 1

Characteristics of hosts and known geographic distribution of recognized and proposed species and strains in the genus Echinococcus

Species strain/isolate Known intermediate hosts Infective to Known definitive hosts Probable geographical distribution
(genotype) Known intermediate hosts

Echinococcus granulosus

Sheep strain (G1)

Sheep, cattle, pigs, Camels, Yes goats, macropods

Tasmanian sheep strain (G2) Buffalo strain (G3)

Horse strain (G4)

Cattle strain (G5)

Camel strain (G6)

Pig strain (G7)

Cervid strain (G8)

Fennoscandinavian cervid strain (G10)

Lion strain

\begin{tabular}{|c|c|c|}
\hline Sheep, cattle? & Yes & Dog, fox \\
\hline Buffalo, cattle? & $?$ & Dog, fox? \\
\hline Horses and other equines & No & Dog \\
\hline Cattle & Yes & Dog \\
\hline Camels, goats, cattle? & Yes & Dog \\
\hline Pigs & Yes & Dog \\
\hline Moose, caribou, reindeer & Yes & Wolf, coyote, dog \\
\hline Reindeer, moose? & ? & Wolf, dog \\
\hline
\end{tabular}

bushpig buffalo,

various antelope, giraffe?

Hippopotamus?

\section{Dog, fox, dingo, jackal and hyena}

Australian mainland, Europe, USA, New Zealand, Africa, China, Middle East, South America and Russian Federation

Tasmania, Argentina

Asia

Europe, Middle East, South Africa

Europe, South Africa, India, Nepal, Russian Federation, South America?

Middle East, Africa, China, Argentina E. granulosus?

Europe, Russian Federation,

South America

North America, Eurasia

Eurasia

Africa
Proposed taxonomic designation

E. granulosus

E. granulosus

E. granulosus

Echinococcus equinus

Echinoccus ortleppi

E. intermedius

E. granulosus

E. granulosus?

E. granulosus?

Echinococcus multilocularis

European isolate
Alaskan isolate
North American isolate
Hokkaido isolate

$\begin{array}{lc}\begin{array}{l}\text { Rodents, domestic and } \\ \text { wild pig, dog, monkey }\end{array} & \text { Yes } \\ \text { Rodents } & \text { Yes } \\ \text { Rodents } & \text { Yes } \\ \text { Rodents, pig monkey, } & \text { Yes } \\ \text { horse } & \end{array}$

\author{
Fox, dog, cat, \\ wolf raccoon-dog \\ Fox, dog, cat \\ Fox, dog, cat, coyote \\ North America \\ Fox, dog, cat raccoon-dog Japan
}

\section{E. multilocularis \\ E. multilocularis \\ E. multilocularis \\ E. multilocularis}

Echinococcus vogeli

(None reported)

Rodents

Yes

Bush dog

Central and South America

E. vogeli

Echinococcus oligarthrus

(None reported)

Rodents

Yes

Wild felids

Central and South America

E. oligarthrus

Echinococcus shiquicus

(None reported)

Rodents, lagomorphs?

Tibetan fox

Tibetan Plateau (China)

E. shiquicus

Modified from Thompson and McManus [1].

drial and genomic DNA of the variant populations provide reliable genetic markers to distinguish them [1-3]. It is likely that the speciation question will be debated for some time and, until it is further clarified, it is important to recognize that important biologic differences exist between populations presently identified in many texts as E. granulosus, the causative agent of cystic echinococcosis, and that these may account for local differences in patterns of transmission and clinical and public health significance of the disease.

\subsection{Cystic echinococcosis (CE)}

In many countries of these endemic regions, national diagnostic incidence rates of $\mathrm{CE}$ range from 5 to 20 per 100,000 population [4]. "National" rates are misleading, however, because most urban populations are at low risk; in rural endemic areas diagnostic incidence is manyfold higher. Furthermore, rates based on clinically diagnosed cases underestimate the burden of infection; surveys of populations in endemic populations using ultrasound imaging diagnostic techniques often measure $\mathrm{CE}$ prevalences of $2 \%$ to $6 \%$, manyfold higher than evident from clinically diagnosed cases [5-7].

Socioeconomic and cultural characteristics are among the best-defined risk factors for human infection with $\mathrm{CE}$ throughout its broad global range: uncontrolled dogs living closely with people, uncontrolled slaughter of livestock, and insanitary living conditions [4]. Whether it be among Arabs in Tunisia, Quechua Indians in Peru, Turkana tribesmen in Kenya, or Tibetans in China these common factors are readily discernible. Consistently highest prevalence is found among populations involved with sheep raising, thus emphasizing the predominant public health importance of the sheep genotype $E$. granulosus (G1). In these regions the social and economic impacts of the infection can be highly significant $[4,8]$.

Although mortality rates associated with hydatid disease may appear low in comparison with those for some other infectious diseases, the morbidity associated with each case is considerable. Patients with hydatid disease often require multiple surgical interventions. Extensive secondary hydatid 
disease often becomes inoperable, and involvement of the bones usually requires amputation. Economic losses to affected families include surgical and hospital expenses as well as loss of income. Published data on the "burden" of echinococcosis from many countries extensively document the severe impact of these infections in terms of their impact on human health and productivity as well as the monetary losses incurred as a result of infection in livestock hosts [8].

Recent investigations in China have demonstrated that, in addition to direct medical costs, the long-term quality of life in patients treated for $\mathrm{CE}$ and $\mathrm{AE}$ was permanently affected indicating that morbidity is more subtle and considerably greater than previously appreciated. Budke and others [9] determined that individuals who were previously unaware they had the disease, but diagnosed with $\mathrm{CE}$ or $\mathrm{AE}$ in a random cross-sectional study using abdominal ultrasound, experienced a significant decrease in the quality of life associated with receiving a positive diagnosis of $\mathrm{CE}$ or $\mathrm{AE}$. Their investigation determined that the DALYs (disability adjusted life years) lost due to these infections in terms of medical treatment costs, lost income, and physical and social suffering, was substantial. Such decreases in the quality of life presumably have significant economic effects including impairment in the work place and thus having less highly paid work than would otherwise have been the case, having greater levels of absenteeism from work, or possibly requiring greater levels of long term nursing care.

Imaging technology developed in recent years has revolutionized detection of hydatid cysts and other space-occupying lesions in all parts of the body. Computed tomography (CT), magnetic resonance imaging (MRI), and ultrasound imaging are all useful for detecting and for defining the extent and condition of avascular fluid-filled cyst(s) in most organs [10]. These noninvasive imaging techniques have proved invaluable for diagnosis and preoperative evaluation by staging the condition of the lesion (intact; unilocular; ruptured; complicated with daughter cysts; and calcified), the extent of the lesion in reference to other organs and vital structures, and identifying the presence of additional, occult lesions. Ultrasonography, because of its relatively low cost and portability, has been used widely for screening, clinical diagnosis, and monitoring of treatment of liver and intra-abdominal cysts [11]. The WHO Informal Working Group on Echinococcosis (WHO-IWGE) proposed a standard ultrasound classification of hydatid cysts based on their ultrasound appearance consistent with the natural history of the disease and response to treatment which is useful for defining the extent and condition of the cyst(s) [12]. The standardized classification scheme is intended to promote uniform standards of diagnosis and treatment and may be applied to clinical management of patients as well as to field diagnostic surveys.

Serologic tests are useful for confirming presumptive imaging diagnoses; however, the limitations of serodiagnosis in cystic echinococcosis must be understood to correctly interpret the findings. Specific confirmation of reactivity can be obtained by demonstrating echinococcal antigens by immunodiffusion (arc 5) procedures or immunoblot assays
(8-, 16-, 21-kDa bands) [13,14]. These latter serodiagnostic markers are the most E. granulosus-specific criteria described, but even they may be detected in serum of patients with other forms of echinococcosis and $5 \%$ to $10 \%$ of patients with $T$. solium cysticercosis [15].

Until recent decades, surgery was the only option for treatment of echinococcal cysts, however, chemotherapy with benzimidazole compounds and, more recently, cyst puncture, and percutaneous aspiration, injection of chemicals, and reaspiration (PAIR) are increasingly seen to supplement or even replace surgery as the preferred treatment. The benefits and limitations of current treatment options have been critically reviewed by consultants for the World Health Organization (WHO) Informal Working Group on Echinococcosis [16].

The development and use of ultrasound imaging technology for clinical diagnosis and population screening has led to significant improvements in clinical management of abdominal forms of echinococcosis and has provided new information on the natural history of the disease; it is now clear that many, if not the majority of cases of CE, have a benign prognosis. This knowledge has led to new, unanswered questions about the necessity for, criteria for, and selection of appropriate treatments. Development of definitive guidelines is still hampered by the lack of a) complete knowledge of the natural history of the disease, b) properly powered clinical trials, and c) adequate long term follow-up. Coordination of effort in longterm multicenter trials will be crucial to significantly improve knowledge and technology of treatment.

E. granulosus has been viewed as highly vulnerable to the implementation of preventive measures, at least in theory. When only synanthropic hosts under human control are involved, the cestode cycle can be interrupted if dogs are prevented from consuming infected viscera from sheep and other domestic ungulates. Indeed, there are now a number of successful examples of echinococcosis control, achieved on a national or regional scale, that confirm that E. granulosus is relatively unstable in synanthropic hosts and responds readily to comprehensive and consistently applied measures of intervention $[4,17]$.

Progress in developing effective vaccination against infection with oncospheres and immunotherapy of the metacestode have been reviewed by Lightowlers and others [18]; vaccination may provide an additional tool for control and prevention of this infection.

\subsection{Alveolar echinococcosis $(A E)$}

Being largely confined to life cycles involving foxes and arvicolid rodents, in ecosystems generally separate from humans, exposure of humans to E. multilocularis is relatively less common than exposure of humans to E. granulosus, the cause of cystic echinococcosis. Fox trappers and other persons who work with foxes or their fur would appear to be relatively frequently exposed to eggs of E. multilocularis, but these occupations have not been associated with higher rates of infection $[19,20]$. There is ecological overlap to humans because domestic dogs or cats may become infected when 
they eat infected wild rodents and infected pets are an important source of infection for humans [21]. Hyperendemic foci have been described in some Native American villages of the North American tundra (now largely disappeared) and in China $[4-6,22]$ where local dogs regularly feed on infected commensal rodent and lagomorph intermediate hosts. Under these circumstances, indirect or direct contamination from feces of infected dogs appears to be the most important source of infection and human infection prevalence may reach $2-6 \%$ $[5,6]$.

E. multilocularis has an extensive geographic range in the Northern Hemisphere, widespread and increasing in Europe, most of northern Eurasia, from Bulgaria and Turkey through most of Russia, and the newly independent nations of the former U.S.S.R., extending eastward to several of the Japanese islands [4]. Recent surveys in Central Europe have extended the known distribution of $E$ multilocularis from four countries at the end of the 1980s to at least 11 countries in 1999 [23]. The incidence of diagnosed disease in humans remains low, between 0.02 and 1.4 per 100,000 for entire countries or larger regions of endemicity. Between 1982 and 2000, 559 cases of alveolar echinococcosis in humans were reported voluntarily from nine European countries to the European Echinococcosis Registry [24]. The cestode appears to have spread eastwards in Europe in association with increased populations of foxes and this may herald the emergence of $\mathrm{AE}$ in humans in central Eastern Europe. Surveys of foxes in Germany and France carried out in the mid-1990s compared recent data with that collected in the 1970s and 1980s, and documented higher fox densities as well as increased prevalences of E. multilocularis infection [25]. New human cases have been reported during the last 10 years in several European countries previously considered non-endemic: i.e. Belgium, Netherlands, Luxembourg, Czech Republic, Slovak Republic, Poland, Italy, Slovenia, Hungary, Bulgaria and Romania [24]. Increasing fox populations, increasing encroachment of foxes into urban areas and spillover of E. multilocularis infection from wild carnivores to domestic dogs and cats might presage increased public health risks of AE [26].

The known distribution and prevalence of infection in foxes and coyotes has increased in the United States and now extends from Montana in the West to western Ohio in the East [27,28]. Exposure of humans appears rare. To date only two persons are known to have acquired their infections in the endemic region in central North America-a 54-year-old man from Manitoba, Canada, and a 60-year-old woman from Minnesota; however, the potential exists for a more serious public health problem [4].

Alveolar echinococcosis closely mimics hepatic carcinoma and is a challenge to diagnose, even in endemic regions. CT and MRI imaging diagnostic criteria have been described [16]. Serologic tests are usually positive at high titers [29] and highly specific antigens [30-32] have been identified and synthesized that, when used in serologic assays, are highly sensitive and specific for diagnosis of $\mathrm{AE}$ and can distinguish this infection from CE (E. granulosus) and other forms of echinococcosis. Antibodies of the IgG1 and IgG4 isotypes are the most sensitive $\operatorname{IgG}$ responses in alveolar echinococcosis and monitoring of these isotypes tended to correlate with active vs. inactive disease and successful treatment [33].

In seronegative patients PCR reactions for detection of echinococcal-specific RNA or DNA, in closed or open biopsy specimens, have been developed and may confirm the diagnosis [34,35].

Clinicians familiar with this disease emphasize that management of alveolar echinococcosis, characterized by a malignant-like proliferating cestode, is extremely difficult and usually requires radical surgery, ideally at an early stage, and long-term to life-long chemotherapy with benzimidazoles [36]. Choosing from the variety of treatment options requires specific clinical experience, therefore, patients should be referred to recognized national or regional alveolar echinococcosis treatment centers [37]. Experience in $\mathrm{AE}$ screening programs in Japan and elsewhere have shown that early diagnosis reduces morbidity and mortality.

Active intervention for control of E. multilocularis presents special difficulties because the primary cycle is almost always sylvatic. In most regions where E. multilocularis is enzootic, controlling the cestode by eliminating its sylvatic hosts would be impractical for economic and logistic reasons or unacceptable for ecological reasons. Where exposure of humans is mainly related to cycles involving synanthropic hosts, that is, dogs, protection might be achieved by regular taeniacidal treatments of dogs. Periodic mass echinococcidal treatments of dogs as an approach to reducing the risk of human infection was evaluated in a 10-year field trial in a village on St. Lawrence Island, Alaska, where E. multilocularis was hyperendemic [38]. All dogs in this village were given praziquantel 5 $\mathrm{mg} / \mathrm{kg}$ body weight at monthly intervals. This was effective in reducing egg contamination as evidenced by an average $83 \%$ reduction in infection prevalence in voles during the course of the trial; prevalence in locally captured voles declined from an average of $29 \%$ at the beginning of the trial to less than $5 \%$ at the end. This control method, which is relatively costly, may be applicable to other regions where synanthropic cycles create a high risk for local human populations; such areas may include recently described endemic foci in Gansu, Qinghai and Sichuan provinces, China, where recent surveys showed high rates of infection in domestic dogs $[5-7,22]$. The costs and benefits of this approach require further evaluation.

\section{Cysticercosis/taeniasis (T. solium)}

Cysticercosis/taeniasis, caused by the "pork tapeworm", $T$. solium, has emerged just as dramatically as echinococcosis. Although commonly recognized by pathologists and neurosurgeons staffing clinics in endemic regions, international emergence of this zoonotic infection required the development of imaging technology [computerized tomographic (CT) and magnetic resonance (MRI) imaging] which permitted the demonstration of viable cysticerci in the brains of infected persons [39]. Prior to the availability of modern diagnostic imaging technology, antemortem diagnosis of cerebral cysticercosis was mostly limited to patients with calcified cysts that 
could be demonstrated in patient's brains by radiographic imaging and, in deceased patients, at autopsy. Today CT and MRI are widely available in hospital settings and the criteria for diagnosis and staging of cysticercosis are described [39,40]. The art and science of immunologic diagnosis of cysticercosis and taeniasis has proceeded rapidly from demonstration of the diagnostically important antigens in larval and adult-stage parasite stages $[41,42]$, assessment of sensitivity and specificity, isolation of the key antigenic components and development of practical diagnostic technology for laboratory and field applications [29,43-46]. Complementing immunologic assays has been development of genetic markers and modification for molecular assays [29,47] These efforts have resulted in a variety of immunologic and molecular diagnostic techniques that can be applied to diagnosis of cysticercosis and taeniasis for clinical and epidemiologic studies.

Deficiencies in diagnostic detection of adult-stage intestinal tapeworm carriers have for long limited epidemiologic studies and control strategies based on detection and treatment of tapeworm carriers. Detection of eggs in feces is insensitive and nonspecific. The development of fecal antigen assays and antibody assays for detection of antibody specific to adult-stage infection provided technology of potential application in epidemiologic surveys and control interventions [29,45-47].

A major factor in improving clinical management of this disease was the development of effective chemotherapy. The efficacy of and strategies for use of praziquantel and albendazole have now been well-described and consensus treatment guidelines have been developed [48-50].

Data obtained from epidemiologic surveys and clinical experiences document that $T$. solium cysticercosis affects millions of people throughout the world and is a major public health problem for most of the developing world [39]. A recent estimate of the prevalence of neurocysticercosis in Latin America indicated that $\sim 400,000$ persons have symptomatic disease and many more are infected but not yet symptomatic [51].

Although T. solium is not transmitted in its swine-human life cycle in the United States, it has dramatically emerged as a common cause of seizures among immigrants from Mexico and other endemic areas $[39,40]$. Diagnoses of neurocysticercosis (NCC) in the United States have followed the movements of migrants in the United States from California north to Oregon and Washington states and west to Texas, the Southeastern and Middle Atlantic states. Although the overwhelming majority of cases of NCC patients have been diagnosed in immigrants who were presumably infected in their countries of origin, foci of autochthonous transmission have been recognized from immigrant Taenia carriers to family members and other contacts $[39,52]$. Similar situations resulting in intrahousehold transmission associated with employment of infected household employees have been documented in Kuwait [53] and Peru [54].

Estimating the global economic impact of cysticercosis is handicapped by inadequate information on the prevalence and public health importance of the disease in most countries. However, the economic losses appear to be highly significant. In Mexico, for example, porcine cysticercosis is responsible for a loss of more than one-half of the national investment in swine production and for more than US\$17 million annually in hospitalization and treatment costs for humans with neurocysticercosis [55]. For all of Latin America, porcine cysticercosis accounts for an estimated economic loss of US\$164 million. $T$. solium taeniasis/cysticercosis is an important example of an imported infection disease which impacts on the health of the United States' population but requires international coordinated efforts to prevent or limit transmission.

In 1993, the International Task Force For Disease Eradication declared T. solium a potentially eradicable infection [56]. The parasite has several characteristics that appear to make it vulnerable to eradication: 1) the life cycle requires humans as definitive hosts, 2) tapeworm infections in humans are the only source of infection for pigs, the natural intermediate hosts, 3) the life span of pigs rarely exceeds 1 year and swineherds can be managed, 4) no reservoirs for infection exist in wildlife, 5) T. solium gradually disappeared from most European countries even without control measures targeted specifically at it. Factors credited with the elimination of $T$. solium include improvements in general sanitation and economic status, the introduction of indoor pig husbandry, and rigorous meat inspection. Despite this historical experience and despite the remarkable recent improvements in technology to diagnose and treat this disease, under the conditions that exist currently in countries where $T$. solium infection is endemic, and given available resources, we do not yet know what is required to reduce and ultimately eliminate transmission.

Although a number of intervention trials based on health education and improved sanitation have demonstrated that transmission of $T$. solium can be inhibited temporarily [45], it has become clear that poverty and ignorance combine to thwart changes in behavior and environment sufficient to permanently limit or prevent transmission. Effective elimination of T. solium will require sustained pressure on the parasite in both human and swine hosts. A regional program in a highly endemic area of Peru has been initiated to evaluate several intervention strategies: mass or selected taeniacidal treatment of humans; cysticercidal treatment of pigs; and vaccination of pigs and/or humans. The outcome of this experience will inform future intervention programs directed against $T$. solium cysticercosis.

\section{Importance of international collaboration}

The remarkable progress in development of technology to diagnose, treat and prevent transmission of echinococcosis and cysticercosis has been greatly facilitated by international collaborations that have promoted sharing of ideas and resources. A network of collaborating groups working on echinococcosis was created through the auspices of the World Health Organization (Informal Working Group on Echinococcosis) and has facilitated the production of guidelines on treatment and control [2]. Informal networks of scientists working on cysticercosis such as the Cysticercosis Working Group in Peru and the Cysticercosis Working Group in Asia [57] are leading the way in progress toward improved diagnosis, treatment and elimination of cysticercosis. 


\section{References}

[1] Thompson RCA, McManus DP. Towards a taxonomic revision of the genus Echinococcus. Trends Parasitol 2002;18:452-7.

[2] Eckert J, Gemmell MA, Meslin F-X, Pawlowski ZS. WHO/OIE Manual on Echinococcosis in Humans and Animals: A Public Health Problem of Global Concern. World Organisation for Animal Health (OIE). Paris: OIE; 2001.

[3] Thompson RCA. Echinococcosis. In: Gillespie SH, Pearson RD, editors. Principles and Practice of Clinical Parasitology. West Sussex: J Wiley \& Sons; 2001. p. 586-612.

[4] Schantz PM, Chai J, Craig PS, Eckert J, Jenkins DJ, Macpherson CNL, et al. Epidemiology and control of hydatid disease. In: Thompson RCA, Lymbery AJ, editors. Echinococcosis and Hydatid Disease. Oxon: CABI publishing, 1995. p. 233-331.

[5] Schantz PM, Wang H, Qiu J, Liu J, Saito E, Emshoff A, et al. Echinococcosis on the Tibetan Plateau: prevalence and risk factors for cystic and alveolar echinococcosis in Tibetan populations in Qinghai Province, China. Parasitology 2003;127:S109-20.

[6] Wang Q, Qiu J, Schantz P, He J, Ito A, Liu F. Investigation of risk factors for development of human hydatidosis among households raising livestock in Tibetan areas of Western Sichuan Province. Chin J Parasit Dis 2001;19:93-6.

[7] Wang Q, Vuitton DA, Qiu J, Giraudoux P, Yongfu X, Schantz PM, et al. Fenced pasture: a possible risk factor for human alveolar echinococcosis in Tibetan pastoral communities of Sichuan, China. Acta Trop 2004; 90:285-93.

[8] Torgerson PR. Economic effects of echinococcosis. Acta Trop 2003;85: $113-8$.

[9] Budke CM, Qiu JM, Zinsstag J, Wang Q, Torgerson PR. Use of disability adjusted life years in the estimation of the disease burden of echinococcosis for a high endemic region of the Tibetan plateau. Am J Trop Med Hyg 2004;71:56-64.

[10] Suwan Z. Sonographic findings in hydatid disease of the liver: Comparison with other imaging methods. Ann Trop Med Parasitol 1996;89:261-5

[11] Macpherson CNL, Bartholomot B, Frider B. Application of ultrasound in diagnosis, treatment, epidemiology, public health and control of Echinococcus granulosus and E. multilocularis. Parasitology 2003;127:S21.

[12] Anon B. International classification of ultrasound images in cystic echincoccosis for application in clinical and field epidemiological settings. Acta Trop 2003;85:253-61.

[13] Maddison SE, Slemenda SB, Schantz PM, Fried JA, Wilson M, Tsang VC. A specific diagnostic antigen of Echinococcus granulosus with an apparent molecular weight of 8 kDa. Am J Trop Med Hyg 1989;40: $377-83$.

[14] Moro PL, Gilman RH, Wilson M, Schantz PM, Verastegui M, Garcia HH, et al. Immunoblot and double diffusion (DD5) tests for hydatid disease cross-react with sera from patients with cysticercosis. Trans R Soc Trop Med Hyg 1992;86:422-3.

[15] Ito A, Ma L, Schantz PM, Gottstein B, Liu YH, Chai JJ, et al. Differential serodiagnosis for cystic and alveolar echinococcosis using fractions of Echinococcus granulosus cyst fluid (antigen B) and E. multilocularis protoscolex (Em18). Am J Trop Med Hyg 1999;60:188-92.

[16] Pawlowski ZS, Vuitton EckertJ, Ammann DA, Kern RW, Craig P, et al. Echinococcosis in humans: clinical aspects, diagnosis and treatment. In: Eckert J, Gemmell MA, Meslin F-X, Pawlowski ZS, editors. WHO/OIE manual on echinococcosis in humans and animals: a public health problem of global concern. World Organisation for Animal Health (OIE). Paris: OIE; 2001. p. 20-99.

[17] Gemmell MA, Schantz PM. Formulating policies for control of Echinococcus granulosus. In: Andersen FL, Ouhelli H, Kachani M, editors. Compendium on cystic echinococcosis in Africa and in Middle Eastern countries with special reference to MoroccoProvo, Utah: Brigham Young University Press; 1997. p. 329-45.

[18] Lightowlers MW, Lawrence SB, Gauci CG, Young J, Ralson MJ, Maas D, et al. Vaccination against hydatidosis using a defined recombinant antigen. Parasite Immunol 1996;18:457-62.
[19] Hildreth MB, Sriram S, Gottstein B, Wilson M, Schantz PM. Failure to identify alveolar echinococcosis in trappers from South Dakota in spite of high prevalence of Echinococcus multilocularis in wild canids. J Parasitol 2000;86:75-7.

[20] Gamble WG, Segal M, Schantz PM, Rausch RL. Alveolar hydatid disease in Minnesota. First human case acquired in the contiguous United States. JAMA 1979;241:904-7.

[21] Rausch RL, Schiller EL. Studies on the helminth fauna of Alaska. XXV. The ecology and public health significance of Echinococcus sibiricensis Rausch and Schiller, 1954, on St. Lawrence Island. Parasitology 1956;46:395-419.

[22] Craig PS, Liu D, Macpherson CNL, Dazhong S, Reynolds D, Barnish G, et al. A large focus of alveolar echinococcosis in central China. Lancet 1992;340:826-31.

[23] Eckert J, Deplazes P. Biological, epidemiological, and clinical aspects of echinococcosis, a zoonosis of increasing concern. Clin Microbiol Rev 2004; 17:107-35.

[24] Kern P, Bardonnet K, Renner E, Auer H, Pawlowski Z, Ammann RW, et al. European Echinococcosis Registry: human alveolar echinococcosis, Europe, 1982-2000. Emerg Infect Dis 2003;9:133-9.

[25] Giraudoux P, Craig PS, Delattre P, Bao G, Bartholomot B, Harraga S, et al. Interactions between landscape changes and host communities can regulate Echinococcus multilocularis transmission. Parasitology 2003; 127:S121-31.

[26] Deplazes P, Hegglin D, Gloor S, Romig T. Wilderness in the city: the urbanization of Echinococcus multilocularis. Trends Parasitol 2004; 20:77-84.

[27] Storandt ST, Kazacos KR. Echinococcus multilocularis identified in Indiana, Ohio, and East-central Illinois. J Parasitol 1993;79:301-5.

[28] Storandt ST, Virchow DR, Dryden MW, Hygnstrom SE, Kazacos KR. Distribution and prevalence of Echinococcus multilocularis in wild predators in Nebraska, Kansas, and Wyoming. J Parasitol 2002;89: $420-1$.

[29] Ito A, Craig PS. Immunodiagnostic and molecular approaches for the detection of taeniid cestode infections. Trends Parasitol 2003;9:377-81.

[30] Gottstein B, Jacquier P, Bresson-Hadni S, Eckert J. Improved primary immunodiagnosis of alveolar echinococcosis in humans by an enzymelinked immunosorbent assay using Em2plus antigen. J Clin Microbiol 1993;31:373-6.

[31] Helbig M, Frosch P, Kern P, Frosch M. Serological differentiation between cystic and alveolar echinococccosis by use of recombinant larval antigens. J Clin Microbiol 1993;31:3211-5.

[32] Ito A, Sako Y, Yamasaki H, Mamuti W, Nakaya K, Nakao M, et al. Development of Em18-immunoblot and Em18-ELISA for specific diagnosis of alveolar echinococcosis. Acta Trop 2003;85:173-82.

[33] Gottstein B, Tschudi K, Eckert J, Ammann R. EM2-ELISA for the followup of the alveolar echinococcosis after complete surgical resection. Trans R Soc Trop Med Hyg 1989;83:389-93.

[34] Kern P, Frosch P, Helbig M, Wechsler JG, Usadel S, Backh K, et al. Diagnosis of Echinococcus multilocularis infection by reverse-transcription polymerase chain reaction. Gastroenterology 1995;109:596-600.

[35] Myjak P, Nahorski W, Pietkiewicz H, von Nickisch-Rosenegk M, Styolaczyk J, Kacprzak E, et al. Molecular confirmation of human alveolar echincoccosis in Poland. Clin Infect Dis 2003;37:e121-5.

[36] Reuter S, Jensen B, Buttenschoen K, Kratzer W, Kern P. Benzimidazoles in the treatment of alveolar echinococcosis: a comparative study and review of the literature. J Antimicrob Chemother 2000;46:451-6.

[37] Buttenschoen K, Schorcht P, Reuter S, Buttenschoen DC, Kern P, Beger HG. Surgical therapy of alveolar echinococcosis and long-term outcome [German]. Chirurg 2001;72:566-72.

[38] Rausch RL, Wilson JF, Schantz PM. A program to reduce the risk of infection by Echinococcus multilocularis: the use of praziquantel to control the cestode in a village in the hyperendemic region of Alaska. Ann Trop Med Parasitol 1990;84:239-50.

[39] Schantz PM, Wilkins PP, Tsang VCW. Immigrants, imaging and immunoblots: the emergence of neurcysticercosis as a significant public health problem. In: Scheld WM, Craig WA, Hughes JM, editors. Emerging Infections 2. Washington, DC: ASM Press; 1998. p. 213-42. 
[40] Shandera WX, Schantz PM, White AC. Taenia solium cysticercosis: the special case of the United States. In: Singh G, Prabhakar S, editors. Taenia solium Cysticercosis. From Basic to Clinical Science. Oxon: CABI Publishing; 2002. p. 139-44.

[41] Gottstein B, Tsang VC, Schantz PM. Demonstration of species-specific and cross-reactive components of Taenia solium metacestode antigens. Am J Trop Med Hyg 1986;35:308-13.

[42] Ito A, Plancarte A, Ma L, Kong Y, Flisser A, Cho SY, et al. Novel antigens for neurocysticercosis: simple method for preparation and evaluation for serodiagnosis. Am J Trop Med Hyg 1998;59:291-4.

[43] Levine MZ, Calderon S, Wilkins PP, Lane WS, Asara JM, Hancock K, et al. Characterization, cloning, and expression of two diagnostic antigens for Taenia solium tapeworm infection. J Parasitol 2004;90:631-8.

[44] Bueno EC, Sheel CM, Vaz AJ, Machado LR, Livramento JA, Takayanagui $\mathrm{OM}$, et al. Application of synthetic $8-\mathrm{kD}$ and recombinant GP50 antigens in the diagnosis of neurocysticercosis by enzyme-linked immunosorbent assay. Am J Trop Med Hyg 2005;72:278-83.

[45] Flisser A, Sarti E, Lightowlers M, Schantz P. Neurocysticercosis: regional status, epidemiology, impact and control measures in the Americas. Acta Trop 2003;87:43-51.

[46] Allan JC, Avila J, Garcia-Noval J, Flisser A. Immunodiagnosis of taeniasis by coproantigen detection. Parasitology 1990;101:473-7.

[47] Gonzalez LM, Montero E, Puente S, Lopez-Velaz R, Harnandez M, Sciutto E, et al. PCR tools for the differential diagnosis of Taenia saginata and Taenia solium taneiasis/cysticercosis from different geographical locations. Diagn Microbiol Infect Dis 2002;42:243-9.

[48] Garcia HH, Pretell EJ, Gilman RH, Martinez SM, Moutton LH, Del Brutto $\mathrm{OH}$, et al. A trial of antiparasitic treatment to reduce the rate of seizures due to cerebral cysticercosis. N Engl J Med 2004;350:249-58.
[49] Garcia HH, Del Brutto OH, Nash TE, White Jr AC, Tsang VC, Gilman RH. New concepts in the diagnosis and management of neurocysticercosis (Taenia solium). Am J Trop Med Hyg 2005;72:3-9.

[50] Garcia HH, Evans CAW, Nash TE, Takayanagui OM, White Jr AC, Botero D, et al. Current consensus guidelines for treatment of neurocysticercosis. Clin Microbiol Rev 2002;15:747-56.

[51] Bern C, Garcia HH, Evans C, Gonzalez AE, Verastegui M, Tsang VC, et al. Magnitude of the disease burden from neurocysticercosis in a developing country. Clin Infect Dis 1999;29:1203-9.

[52] Schantz PM, Moore JL, Munoz BJ, Hartman BJ, Schaefer JA, et al. Neurocysticercosis in an Orthodox Jewish community in New York city. New Engl J Med 1992;327:692-5.

[53] Hira PR, Francis I, Abdella NA, Gupta R, Ai-Ali FM, Grover S, et al. Cysticercosis: imported and autochthonous infections in Kuwait. Trans R Soc Trop Med Hyg 2004;98:233-9.

[54] Huisa BN, Menacho LA, Rodriguez S, Bustos JA, Gilman RH, Tsang VC, et al. Taeniasis and cisticercosis in housemaids working in affluent neighborhoods in Lima, Peru. Am J Trop Med Hyg 2005;73:496-500.

[55] Carabin H, Budke CM, Cowan LD, Willingham AL, Torgerson PR. Methods for assessing the burden of parasitic zoonoses: echincoccosis and cysticercosis. Trends Parasitol 2005;21:327-33.

[56] Schantz PM, Cruz M, Sarti E, Pawlowski Z. Potential eradicability of taeniasis and cysticercosis. Bull Pan Am Health Organ 1993;27:397-403.

[57] Ito A, Wandra T, Yamasaki H, Nakao M, Sako Y, Nakaya K, et al. Cysticercosis/taeniasis in Asia and the Pacific. Vector-Borne Zoonotic Dis 2004;4:95-107. 